\title{
Investigation of the effect of latent toxoplasmosis on oxidative stress in acne vulgaris patients
}

\author{
Original Alshaimaa MR Hamed ${ }^{1}$, Marwa S El-Mesidy ${ }^{2}$, Eman H Elsebaei ${ }^{3}$, Amal M \\ Article $\quad$ Mahfoz $^{4}$, Magda SA Abdeltawab ${ }^{1}$
}

Departments of Medical Parasitology ${ }^{1}$, Dermatology ${ }^{2}$, Public Health and Community Medicine $^{3}$, and Pharmacology and Toxicology ${ }^{4}$, Faculty of Medicine, Cairo University ${ }^{1-3}$, and Faculty of Pharmacy, Modern University for Technology and Information ${ }^{4}$, Cairo, Egypt

\begin{abstract}
Background: Acne vulgaris is a common skin condition that plagues many individuals and reflects on their quality of life. The pathogenesis of acne is multifactorial and is subject of continuous investigation and research. Infection, inflammation, and oxidative stress are among the numerous theories explaining the pathogenesis of acne. Meanwhile, the etiological factors of acne vulgaris share common grounds with immune alterations during toxoplasmosis.

Objectives: Investigation of a possible association between toxoplasmosis and the pathogenesis of acne vulgaris.

Subjects and Methods: Serum anti-Toxoplasma IgG and glutathione (GSH) were measured in 43 acne patients and 40 healthy control subjects. Serological detection of anti-Toxoplasma IgG was performed using enzyme linked immunosorbent assay (ELISA) technique and GSH level in serum was detected using colorimetry. In addition, clinical evaluation of the acne lesions was assessed.

Results: GSH was significantly lower in seropositive acne patients as compared to seronegative ones. No difference in seroprevalence and anti-Toxoplasma IgG titre was observed between cases and controls. Clinical evaluation of cases revealed that patients with severe acne showed a higher Cardiff acne disability index (CADI) score.

Conclusion: The reduction of GSH in seropositive cases suggests a contributing role of toxoplasmosis to the development of acne by altering the oxidative balance. The involvement of toxoplasmosis in the pathogenesis of multi-factorial clinical conditions such as acne vulgaris should be focused upon since latent infection is widely present among the Egyptian population.
\end{abstract}

Keywords: acne, GSH, toxoplasmosis, severity score.

Received: 26 September, 2020, Accepted: 27 October, 2020.

Corresponding Author: Alshaimaa MR Hamed, Tel.: +20 1026893667, E-mail: amsolaiman@kasralainy.edu.eg

Print ISSN: 1687-7942, Online ISSN: 2090-2646, Vol. 13, No. 3, December, 2020.

\section{INTRODUCTION}

Toxoplasma gondii is an obligate intracellular protozoon, that affects about $30 \%$ of the population all over the globe ${ }^{[1]}$. Although it is considered as one of the most widespread disease agents, clinical diseases caused by this coccidial parasite are not as common, since the majority of infected persons remain asymptomatic ${ }^{[2]}$. Toxoplasmosis exists mainly in its latent form, with reactivation of infection occurring in immunocompromised persons, since $T$. gondii is an opportunistic parasite. Acute toxoplasmosis is often underdiagnosed due to the ambiguity of clinical symptoms such as lymphadenitis and sore throat ${ }^{[3]}$. Since T. gondii is an intracellular parasite, it reaches many body systems and has a wide range of effects on the host immune system. Toxoplasmosis has also been found to have an impact on the oxidative status of the host, where markers of oxidative stress such as increased malondialdehyde (MDA) levels and reduced GSH activity were found to be associated with antiToxoplasma seropositivity ${ }^{[4]}$.
This wide spectrum of physiological alterations observed concomitantly with toxoplasmosis suggests the possible involvement of this protozoal infection in multifactorial disorders attributed to immuneendocrine alterations. One of these disorders is acne vulgaris, which is an inflammatory skin condition appearing in late adolescence ${ }^{[5]}$. Oxidative stress plays a role in the pathogenesis of acne in part due to the generation of reactive oxygen species (ROS) in response to infection by the bacterium Propiono bacterium acnes, which colonizes the skin and grows in plugged hair follicles, thus attracting inflammatory cells, mainly neutrophils. These in turn secrete inflammatory mediators and generate ROS, which augments the inflammatory response and tissue damage ${ }^{[6]}$. Since the etiological factors of acne vulgaris share common grounds with immune alterations occurring during toxoplasmosis, a possible association between this protozoal infection and the pathogenesis of acne should be investigated. 
The current study aims at investigating the existence of this association by measuring serum levels of GSH in acne vulgaris patients, with serological evidence for the presence or absence of toxoplasmosis.

\section{SUBJECTS AND METHODS}

Study subjects and setting: The current study is a case-control analytical study, in which venous blood samples were collected from 43 acne vulgaris patients (age range 15-55 years) attending the Kasr Al Ainy Dermatology Outpatient Clinic, Cairo University, Egypt. Forty healthy individuals not suffering from acne were considered as control subjects. Inclusion criteria include both male and female cooperative patients. Exclusion criteria include patients suffering from disease conditions related to toxoplasmosis other than acne or having been previously diagnosed as suffering from toxoplasmosis, or having previously received anti-Toxoplasma therapy, or badly collected samples.

Study parameters: Data collection was performed by a specialized dermatologist covering socio-demographic and clinical data. Serological anti-Toxoplasma IgG was detected using ELISA ${ }^{[7]}$, while GSH level in serum was detected using colorimetry ${ }^{[8]}$.

Specimen collection and preparation: Venous blood samples $(5 \mathrm{ml})$ were drawn under aseptic conditions into sterile blood tubes without additives. Collected blood samples were centrifuged at 3,000 rpm for $5 \mathrm{~min}$ for sera preparation and separation. Prepared serum samples were coded and stored at $-20^{\circ} \mathrm{C}$ until tested.

Clinical evaluation of patients: In addition to the socio-demographic and clinical data, severity scaling using the global acne severity score (GEA Scale) ${ }^{[9]}$, as well as the assessment of the quality of life using the CADI were performed ${ }^{[10]}$. The GEA scale depends on the assessment of the dominant lesions, the degree of inflammation and the area of skin involvement. The CADI is a 5-item questionnaire concerned with evaluating the emotional and social impact of acne lesions on the quality of life of patients. Its score ranges from $0-15$. Also, the skin type of patients was evaluated in accordance to the Fitzpatrick skin type classification ${ }^{[11]}$. This grading system classifies the skin type from 1-6 according to the degree of melanin pigmentation in the skin where the fairest skin is designed as type 1 , while the dark brown skin is designed as type 6 .

ELISA for the detection of anti-Toxoplasma IgG in serum ${ }^{[7]}$ : The assay was performed and evaluated according to the manufacturer's instructions provided in the Toxoplasma IgG ELISA CALBIOTECH test kit supplied by Beta Lab, USA. Diluted patients' sera were added to wells coated with purified Toxoplasma antigen. Anti-Toxoplasma IgG specific antibody, if present, bounded to the antigen. All unbound material was washed away, and the enzyme conjugate was added to bind to the antibody- antigen complex, if present. Excess enzyme conjugate was washed off and a substrate was added. The intensity of the colour generated was proportional to the amount of IgG antibody in the sample. Reading the absorbance of each sample on an ELISA reader was done at a wavelength of $450 \mathrm{~nm}$. The dual wavelength was measured with a reference filter at $620 \mathrm{~nm}$. Calculation of the sample titre was done according to the following equation: Antibody titre $=$ absorbance of each well/cut off value Cut off value $=$ optical density of the calibrator (1.908) multiplied by the calibrator factor on calibrator bottle (0.35). Results were interpreted as follows: $<0.9=$ negative; $0.9-1.1$ = borderline $;<1.1$ = positive .

Colorimetric method for estimation of serum GSH $^{[8]}$ : This was performed as described in the reduced GSH kit provided by BIOACTIVIA DIAGNOSTIC, Egypt. The method is based on the reduction of 5,5' dithiobis (2-nitrobenzoic acid) (DTNB) with GSH to produce a yellow compound. The reduced chromogen was directly proportional to the GSH concentration and its absorbance was measured at $405 \mathrm{~nm}$. The calculation of GSH was expressed in $\mathrm{mmol} / \mathrm{L}$. Reference value stated in the kit was $4 \mathrm{mmol} / \mathrm{L}$.

Statistical analysis: Microsoft excel 2013 was used for data entry and the statistical package for social science (SPSS) version 24 (SPSS, Armonk, New York: International Business Machines Corporation) was used for data analysis. Simple descriptive statistics (arithmetic mean and standard deviation) were used for the summarization of quantitative data and frequencies were used for qualitative data. T-independent and one-way ANOVA were used to compare normally distributed quantitative data. The level of significance was set at probability $(P)$ value $<0.05^{[12]}$.

Ethical considerations: The study was conducted following international guidelines and in accordance with the ethical guidelines of the Faculty of Medicine, Cairo University. Informed consent was obtained from all patients included in this study.

\section{RESULTS}

In the current study, 43 patients suffering from acne vulgaris were included, while 40 individuals free from acne were considered as control subjects. Table (1) presents the distribution of personal and clinical data among the patients involved in this study. Patients comprised 13 males (30.2\%) and 30 females $(69.2 \%)$. They were mostly single $(81.4 \%)$, only $18.6 \%$ were married. Students were largely represented by $51.2 \%$, while $20.9 \%$ were employed and $27.9 \%$ were unemployed. The age range of the patients was $15-20$ years in $53.5 \%$ and $21-30$ years in $46.5 \%$. Thirteen patients $(30.2 \%)$ were suffering 
from acne for more than five years, and 16 patients (37.2\%) for 2-5 years, whereas 14 patients (32.6\%) had a recent history of less than 1 year. Clinical evaluation of patients revealed that the majority had type 3 or type 4 skin types (44.19\% each). No patients had type 1 skin, 2 patients had type 2 skin $(4.65 \%)$ and 3 patients had type 5 skin $(6.98 \%)$.

The severity of acne lesions as assessed by the GEA severity scale for evaluation of lesion dominance, extent, and inflammation was mild in 22 patients $(51.2 \%)$, moderate in 12 patients $(27.9 \%)$ and severe in 9 patients (20.9\%). Assessment of acne-related disability by the CADI score showed that $27.9 \%$ of patients had a score of $<5,26.6 \%$ patients had a score between $6-10$, while $46.5 \%$ of patients had a score between 11-15 (Table 1).

\section{Relation between GSH and Toxoplasma} seropositivity: GSH levels were lower in seropositive patients $(3.0 \pm 0.9)$ compared to the seronegative ones $(5.1 \pm 1.2)$ with statistically significant values $(P<0.0001)$, indicating that the level of oxidative stress is significantly higher in Toxoplasma seropositive individuals than seronegative ones (Figure 1).

Relation between GSH and acne: GSH levels were lower in acne cases $(4.05 \pm 1.48)$ as compared to controls $(4.42 \pm 0.72)$ with no statistical significance $(P=0.37)$ (Figure 1).

Relation between Toxoplasma and acne: Twentyone patients (48.8\%) suffering from acne were found to be seropositive for toxoplasmosis as compared to $18(45.0 \%)$ control subjects. The anti-Toxoplamsa IgG titre was apparently higher in acne cases $(1.30 \pm 1.19)$ than controls $(0.93 \pm 1.09)$ (Table 2$)$, with no statistical significance between cases and control subjects in seroprevalence or titre $(P=0.726$, and 0.273 , respectively). The mean anti-Toxoplamsa IgG titre in positive cases was $2.53 \pm 0.80$ as opposed to $2.25 \pm 0.75$ in controls, with no statistical significance between the two values $(P=0.259)$ (Table 2).

Table 1. Descriptive personal and clinical data analysis of cases.

\begin{tabular}{|c|c|c|c|c|}
\hline Cases & & & $3(100 \%)$ & \\
\hline Gender & $\begin{array}{c}\text { Male } \\
13(30.2 \%)\end{array}$ & $\begin{array}{c}\text { Female } \\
30(69.2 \%)\end{array}$ & & \\
\hline Marital status & $\begin{array}{c}\text { Single } \\
35(81.4 \%)\end{array}$ & $\begin{array}{l}\text { Married } \\
8(18.6 \%)\end{array}$ & & \\
\hline Employment status & $\begin{array}{c}\text { Student } \\
22(51.2 \%)\end{array}$ & $\begin{array}{c}\text { Employed } \\
9(20.9 \%)\end{array}$ & $\begin{array}{c}\text { Unemployed } \\
12(27.9 \%)\end{array}$ & \\
\hline Age & $\begin{array}{l}\text { 15-20 years } \\
23(53.5 \%)\end{array}$ & $\begin{array}{c}21-30 \text { years } \\
20(46.5 \%)\end{array}$ & & \\
\hline Duration of the disease & $\begin{array}{c}<1 \text { year } \\
14(32.6 \%)\end{array}$ & $\begin{array}{l}2-5 \text { years } \\
16(37.2 \%)\end{array}$ & $\begin{array}{c}\text { More than } 5 \text { years } \\
13(30.2 \%)\end{array}$ & \\
\hline Skin type & $\begin{array}{c}\text { Type } 2 \\
2(4.65 \%)\end{array}$ & $\begin{array}{c}\text { Type } 3 \\
19(44.19 \%)\end{array}$ & $\begin{array}{c}\text { Type } 4 \\
19(44.19 \%)\end{array}$ & $\begin{array}{c}\text { Type } 5 \\
3(6.97 \%)\end{array}$ \\
\hline GEA scale & $\begin{array}{c}\text { Mild } \\
22(51.2 \%)\end{array}$ & $\begin{array}{l}\text { Moderate } \\
12(27.9 \%)\end{array}$ & $\begin{array}{c}\text { Severe } \\
9(20.9 \%)\end{array}$ & \\
\hline CADI scale & $\begin{array}{c}<5 \\
12(27.9 \%)\end{array}$ & $\begin{array}{c}\mathbf{6}-\mathbf{1 0} \\
11(26.6 \%)\end{array}$ & $\begin{array}{c}11-\mathbf{1 5} \\
20(46.5 \%)\end{array}$ & \\
\hline
\end{tabular}

GEA scale: Global acne severity score, CADI scale: Impact of acne lesions on the quality of life.

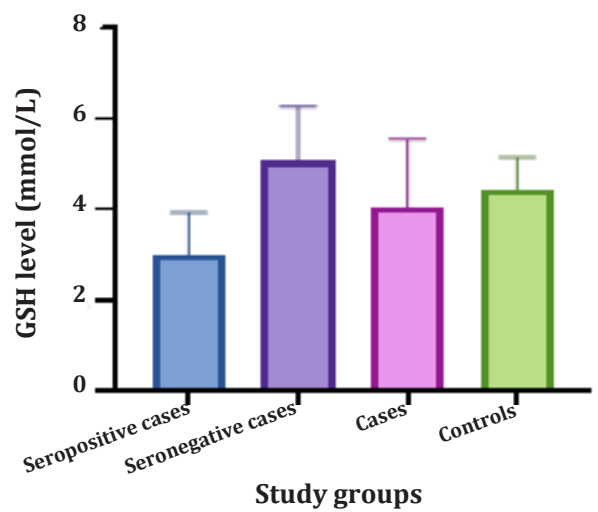

Fig. 1. Column chart showing GSH level (mmol/L) in the different study groups. 


\begin{tabular}{|c|c|c|c|c|}
\hline & $\begin{array}{c}\text { Cases } \\
\text { No. (\%) }\end{array}$ & $\begin{array}{l}\text { Controls } \\
\text { No. (\%) }\end{array}$ & Total & $P$ value \\
\hline \multicolumn{5}{|l|}{ Seroprevalence } \\
\hline Seropositive & $21(48.8 \%)$ & $18(45.0 \%)$ & 39 & \multirow{3}{*}{$0.726^{*}$} \\
\hline Seronegative & $22(51.2 \%)$ & $22(55.0 \%)$ & 44 & \\
\hline \multirow[t]{2}{*}{ Total } & $43(100 \%)$ & $40(100 \%)$ & 83 & \\
\hline & $\begin{array}{c}\text { Cases } \\
\text { Mean } \pm \text { SD }\end{array}$ & $\begin{array}{c}\text { Controls } \\
\text { Mean } \pm \text { SD }\end{array}$ & Total & $P$ value \\
\hline \multicolumn{5}{|l|}{ Anti-Toxoplasma IgG titre ${ }^{@}$} \\
\hline Seropositive only & $2.53 \pm 0.80$ & $2.25 \pm 0.75$ & 39 & $0.259 *$ \\
\hline Seropositive and seronegative & $1.30 \pm 1.19$ & $0.93 \pm 1.09$ & 83 & $0.273^{*}$ \\
\hline
\end{tabular}

@: Titre is below ELISA cut off value. *No statistically significant difference between cases and control subjects at $P<0.05$.

Evaluation of different study parameters in relation to severity of acne: The seropositive group included $12(57.1 \%)$ patients with mild acne, $4(19 \%)$ patients with moderate acne and $5(23.8 \%)$ with severe acne. Patients with severe acne had a significantly higher CADI score $(14.44 \pm 1.67 ; P$ $<0.001$ ). Comparison between anti-Toxoplasma IgG titer and GSH in seropositive patients with different severity grades of acne revealed no significant association (Table 3 ). The CADI score in seropositive acne patients was higher $(11.29 \pm 4.30)$ than that in seronegative ones $(9.41 \pm 4.92)$, with no statistical significance $(P=0.190)$ (Table 4$)$.

Table (3). Anti-Toxoplasma titre, GSH level, and CADI score in relation to GEA scale of seropositive patients.

\begin{tabular}{lcccc}
\hline \hline & Mild & Moderate & Severe & Pvalue \\
\hline Anti-Toxoplasma titre & $1.64 \pm 1.37$ & $0.99 \pm 1.18$ & $1.42 \pm 0.97$ & 0.369 \\
GSH (mmol/L) & $3.92 \pm 1.51$ & $4.33 \pm 1.66$ & $3.97 \pm 1.38$ & 0.779 \\
CADI (0-15) & $9.14 \pm 4.95$ & $9.42 \pm 4.10$ & $14.44 \pm 1.67$ & $<0.001^{*}$ \\
\hline
\end{tabular}

*Statistical significance at $P<0.05$; one-way ANOVA

GEA scale: Global acne severity score, GSH: Glutathione, CADI scale: Impact of acne lesions on the quality of life.

Table (4). CADI score according to sero-reactivity to Toxoplasma antibodies.

\begin{tabular}{lccc}
\hline \hline Sero-reactivity to toxoplasmosis & No. & Mean \pm SD & $P$ value \\
\hline Seropositive & 21 & $11.29 \pm 4.30$ & $0.190^{*}$ \\
Seronegative & 22 & $9.41 \pm 4.92$ & \\
\hline
\end{tabular}

$* \overline{\bar{N} \text { No statistically significant difference between cases and control subjects at } P=0.190}$

\section{DISCUSSION}

Toxoplasmosis is a widespread protozoal infection that has a wide and diverse clinical spectrum. Latent toxoplasmosis was found to reflect on the immune status of the host ${ }^{[4]}$. Acne vulgaris is the most prevalent skin condition which may indicate an underlying and unidentified systemic pathology ${ }^{[13]}$. It may be suggestive of hidden stress, hormonal imbalance, infection, and immune alterations. In the current study, we investigated the possible association between toxoplasmosis and acne since both diseases are reported to involve biochemical alterations such as oxidative stress. Cutaneous toxoplasmosis is generally a rare presentation and is usually found in immune-compromised persons. Fong et $a l^{[14]}$ recounted a case report of painful nodular lesions in an HIV-positive patient. The lesions were found to be positive for toxoplasmosis by electron microscopy and PCR. Zimmermann et al. ${ }^{[15]}$ also published the case report of a patient with aplastic anaemia suffering from varicella-like vesicular lesions. Skin biopsy from these skin lesions led to the diagnosis of toxoplasmosis and was confirmed by immunohistochemistry and PCR. On the other hand, a case of cutaneous toxoplasmosis was reported by Marina et al. ${ }^{[16]}$ in an immune-competent patient presenting with multiple erythematous papules. In our study, we investigated the presence of an association between acne vulgaris and toxoplasmosis by detecting anti-Toxoplasma IgG in acne patients as compared to acne-free control subjects. Acne cases were apparently higher in seropositivity and in titre, however there was no statistically significant difference between both cases and control groups.

We further investigated a possible indirect effect of toxoplasmosis on the development of acne vulgaris by affecting the oxidative state of the 
host. GSH levels in our study were lower in patients than controls with no statistical significance. It was interesting to observe that GSH levels were significantly lower in seropositive patients suffering from acne as compared to seronegative patients. Thus, it is possible to assume that toxoplasmosis does not induce acne per se, but may contribute to the pathogenesis through the generation of oxidative stress, which is reported to be one of the mechanisms of Toxoplasma-induced pathology ${ }^{[17]}$. In parallel, the generation of reactive oxygen species (ROS) by neutrophils also plays a role in the pathogenesis of acne. ROS cause inflammation and damage to the wall of hair follicles leading to the release of squalene peroxides which decreases the level of $\mathrm{GSH}^{[5]}$. Al-Shobaili ${ }^{[18]}$ measured serum levels of oxidative stress biomarkers in acne patients with different degrees of severity. Acne patients had significantly higher MDA levels and a lower total antioxidant capacity than control subjects. Severe grades of acne showed a greater burden of oxidative stress. In a study on superoxide dismutase (SOD) activity and MDA in 23 acne patients, Abdel Fattah et $a l^{[19]}$ did not detect significant difference between patients and control subjects. However, they found that patients with moderate and severe acne had a lower SOD activity and higher MDA levels than patients with mild acne. The implication of oxidative stress in the development of acne vulgaris underlines the role of antioxidants in disease management. Sahib et al. ${ }^{[20]}$ stated that the administration of oral antioxidants in the form Silymarin, Selenium and N-acetylcysteine decreased the severity of acne lesions and improved the oxidative status of patients as indicated by serum oxidative stress biomarkers.

In the study at hand, the severity of acne lesions was assessed by the GEA Scale and its impact on the quality of life was evaluated by the CADI. The latter was significantly higher with the increased degree of severity in lesions. Seropositivity to toxoplasmosis and serum GSH levels, however, did not differ significantly with different grades of lesion severity. Kokandi ${ }^{[21]}$ evaluated the severity of acne in female patients on CADI, but found no significant association. In contrast, Saka et al. ${ }^{[22]}$ evaluated the impact of acne with variable degrees of severity on the quality of life of the patients using the Echelle de Cotation des Lésions d'acné (ECLA) and CADI scores. They concluded that the severity of the disease had a negative impact on the quality of life of the patients.

In conclusion, we did not find an association between seropositivity or sero-intensity of toxoplasmosis with acne, however, a positive association between reduced GSH levels and seropositivity of Toxoplasma in acne patients was detected. This could be indicative of a contributing rather than causative role of toxoplasmosis in the pathogenesis of acne vulgaris through its effect on the status of oxidative stress of the host. Moreover, the involvement of toxoplasmosis in the pathogenesis of multi-factorial clinical conditions such as acne vulgaris should be stressed since latent infection is widely present among the population. Also, the role of antioxidants in the treatment of acne vulgaris should thus be emphasized. Finally, the effect of the severity of acne lesions on the quality of life of the patients, as assessed by the CADI score, mirrors the psychological burden imposed by this common skin condition.

Authors contribution: Hamed AMR drafted the study plan, supplied the essential reagents, collected the samples, drafted the methodology, interpreted the results, and reviewed the manuscript; El-Mesidy MS clinically assessed the patients, scored the disease, recorded the data sheet and reviewed the manuscript; Mahfoz AM supplied the essential tools, assessed laboratory study parameters, evaluated the results and shared in reviewing of manuscript; Elsebaei EH performed the statistical analysis and interpreted the study results; Abdeltawab MSA generated the research idea, drafted the study plan, shared in data analysis and interpretation, drafted and reviewed the writing of paper. All authors critically revised the manuscript and approved the final manuscript.

Declaration of interest: None.

Funding statement: This research did not receive any specific grant from funding agencies in the public, commercial, or not-for-profit sectors.

\section{REFERENCES}

1. Abdoli A, Dalimi A. Are there any relationships between latent Toxoplasma gondii infection, testosterone elevation, and risk of autism spectrum disorder? Front Behav Neurosci 2014; 8: 339.

2. Robert-Gangneux F, Dardé ML. Epidemiology of and diagnostic strategies for toxoplasmosis. Clin Microbiol Rev 2012; 25(2):264-296.

3. Zghair KH, Al-Qadhi BN, Mahmood SH. The effect of toxoplasmosis on the level of some sex hormones in males blood donors in Baghdad. J Parasit Dis 2015; 39(3):393-400.

4. Karaman U, Celik T, Kiran T, Colak C, Daldal NU. Malondialdehyde, glutathione, and nitric oxide levels in Toxoplasma gondii seropositive patients. Korean J Parasitol 2008; 46(4):293-295.

5. Wong A, Zhang B, Jiang M, Gong E, Zhang Y, Lee SW. Oxidative stress in acne vulgaris. J Clin Dermatol Ther 2016; 3: 020.

6. Akamatsu H, Horio T, Hattori K. Increased hydrogen peroxide generation by neutrophils from patients with acne inflammation. Int J Dermatol 2003; 42: 366-369.

7. Fallahi E, Badparva M, Mohammadi M, Ebrahimzadeh F, Pournia Y. Sero-epidemiological study of Toxoplasma gondii in women referred to Khorramabad laboratory of health center for medical examination before marriage, Lorestan Province, Iran. Asian J Biol Sci 2009; 2 (3): 88-94. 
8. Ni P, Sun Y, Dai H, Hu J, Jiang S, Wang Y, et al. Highly sensitive and selective colorimetric detection of glutathione based on $\mathrm{Ag}$ [I] ion-3,3',5,5'tetramethylbenzidine (TMB). Biosens Bioelectron 2015;63:47-52.

9. Dreno B, Poli F, Pawin H, Beylot C, Faure M, Chivot $\mathrm{M}$, et al. Development and evaluation of a global acne severity scale (GEA Scale) suitable for France and Europe. J Eur Acad Drtmatol Venereol 2011; 25 (1): 43-48.

10. Motley RJ, Finlay AY. How much disability is caused by acne? Clin Exp Dermatol 1989; 14: 194-198.

11. Fitzpatrick TB. The validity and practicality of sunreactive skin types I through VI. Arch Dermatol 1988;124(6):869-71.

12. Dawson B, Trapp R. Basic and clinical biostatistics, 4th Edition, New York, United States, Mcgraw-Hill Medical Publishing Inc 2004; pp: 23, 61, 245.

13. Franik G, Bizon A, Włoch S, Kowalczyk K, BiernackaBartnik A, Madej P. Hormonal and metabolic aspects of acne vulgaris in women with polycystic ovary syndrome. Eur Rev Med Pharmacol Sci 2018; (22): 4411-4418.

14. Fong MY, Wong KT, Rohela M, Tan LH, Adeeba K, Lee YY, et al. Unusual manifestation of cutaneous toxoplasmosis in a HIV-positive patient. Trop Biomed 2010; 27(3):447-450.

15. Zimmermann S, Hadaschik E, Dalpke A, Hassel JC, Ajzenberg D, Tenner-Racz $\mathrm{K}$, et al. Varicella-like cutaneous toxoplasmosis in a patient with aplastic anemia. J Clin Microbiol 2013; 51(4):1341-1344.

16. Marina S, Broshtilova V, Botev I, Guleva D, Hadzhiivancheva $\mathrm{M}$, Nikolova $\mathrm{A}$, et al. Cutaneous manifestations of toxoplasmosis: a case report. Serbian J Dermatology Venereol 2014; 6 (3): 113-119.

17. Dincel GC, Atmaca HT. Role of oxidative stress in the pathophysiology of Toxoplasma gondii infection. Int J Immunopathol Pharmacol 2016; 29(2): 226 -240.

18. Al-Shobaili HA. Oxidants and anti-oxidants status in acne vulgaris patients with varying severity. Ann Clin Lab Sci Spring 2014; 44(2):202-207.

19. Abdel Fattah NS, Shaheen M, Ebrahim A, El Okda E. Tissue and blood superoxide dismutase activities and malondialdehyde levels in different clinical severities of acne vulgaris. Br J Dermatol 2008; 159:1086-1091

20. Sahib AS, Al-Anbari HH, Salih M, Abdullah F. Effects of Oral antioxidants on lesion counts associated with oxidative stress and inflammation in patients with papulopustular acne. J Clin Exp Dermatol Res 2012; 03(05).

21. Kokandi A. Evaluation of acne quality of life and clinical severity in acne female adults. Dermatol Res Pract 2010;410809.

22. Saka B, Akakpo AS, Téclessou JN, Mouhari-Toure A, Mahamadou G, Gnossike G, et al. Acne in Lomé, Togo: clinical aspects and quality of life of patients. BMC Dermatol 2018; 18(1):7. 